\title{
Anatomical Considerations in Implant Selection and Positioning
}

D Krishna Prasad, Manoj Shetty, Divya Rajan Mehra

\begin{abstract}
This article reviews the various anatomical facts significant for preoperative planning of implant procedures in the mandible and the maxilla. This planning includes the precise evaluation of distinct anatomical factors, such as the position of the mandibular canal, the maxillary sinus, the width of the cortical plates, the existing bone density, appropriate implant selection and planning the most appropriate implant position in the existing clinical condition.
\end{abstract}

Keywords: Anatomical considerations, Implant placement, Implant selection.

How to cite this article: Prasad DK, Shetty M, Mehra DR. Anatomical Considerations in Implant Selection and Positioning. Int J Oral Implantol Clin Res 2013;4(1):24-29.

\section{Source of support: Nil}

Conflict of interest: None declared

\section{INTRODUCTION}

In the present era, when it comes to oral rehabilitation, a wide array of options are available to restore the missing teeth using the fixed or the removable prosthesis. The advent of implants in the field of dentistry, has given the dental professionals a viable option to provide the patient with nearly a third set of dentition, but, as it is rightly said, 'With great powers, comes greater responsibility’. Today, in dental institutions as well as in private clinical practice oral implants are routinely placed with high success rates. However, this has also increased the incidence of neurosensory disturbances and hemorrhages along with many other complications. It is essential of have a sound knowledge of the anatomy and its variations prior to implant placement to ensure a precise surgical procedure and safeguard the patient against iatrogenic complications. Besides, the availability of options regarding implant dimensions and morphologies have also greatly expanded over the past few years. Hence, appropriate selection of the implant to provide an ideal platform to restore esthetics and function is a must.

\section{Anatomical Considerations for Implant Placement}

A thorough knowledge and information about the adjacent anatomical structures is essential for planning and placement of dental implants. ${ }^{1}$ The available height and width of the residual alveolar bone is crucial for implant placement in both the maxilla and the mandible. Insufficient residual alveolar bone may result in a suboptimal implant placement and subsequent failure of the implant. However, available bone in excessive amounts is also not a conducive clinical situation to place implants as it could create occlusal plane interferences in the completed restoration. In addition to adequacy of available bone, a balance between the cortical and trabecular bone is equally desired. ${ }^{2}$ Too much of cortical bone can delay osseointegration while excess of trabecular bone may limit the early stability of the implant. ${ }^{2}$

Presence of bony undercuts may result into perforation of the cortical bone. Spacing of the implants is another important factor to be borne in mind. Close proximity of the proposed osteotomy site to the apices of the adjacent roots can add to the complications. For proper integration and tissue health, it is recommended that there must be $3 \mathrm{~mm}$ of space between two implants and between teeth and implants. Thus, the space available to place two implants of $4 \mathrm{~mm}$ diameter each, between natural teeth must be around $17 \mathrm{~mm}^{2}$

There are specific anatomical considerations to be considered for maxillary and mandibular arch.

\section{Maxillary Arch}

Important anatomical structures in the maxilla that can pose for complications following implant placement include the nasal floor anteriorly and the maxillary sinus posteriorly. Implant placement in the posterior maxillary region is particularly challenging when compared to the anterior region. Iaotrogenic sinus perforation is a commonly encountered complication when the selected implant length is more than the available bone height in the posterior maxilla. This has been found to be a potential cause for implant failure in the posterior maxilla. ${ }^{3}$ This can be taken care of by selecting a short implant. If a standard length implant is needed to be used in such situation, bone augmentation procedure may be done to increase the height and volume of the bone.

Pneumatization of the maxillary sinus may be seen if the resorption of the bone has occurred from the internal aspect of the sinus walls. This may either occur with progressing age or following tooth loss. In such cases, external/internal sinus lift procedure may be needed before 
implant placement to prevent penetration or perforation of the sinus wall. ${ }^{4,5}$

The sinus lift procedure, developed in the mid 1970s, has been refined and is now frequently performed. A mucoperiosteal flap is created along the anterior wall of the maxillary sinus similar to the Caldwell-Luc approach. A rectangular osteotomy is then cut into the lateral antral wall with the inferior horizontal segment of the rectangle 3 to $4 \mathrm{~mm}$ above the floor of the sinus. The superior horizontal segment of the rectangle is formed by drilling closely positioned holes. This creates a trapdoor, which will be fractured inward and hinged along the superior aspect of the rectangle. The infracturing is done carefully to prevent tearing of the schneiderian (maxillary sinus) membrane. The membrane at the inferior aspect of the osteotomy is dissected from the floor of the maxillary sinus and elevated upward to create a space in the floor of the sinus for the bone-graft material.

Bone-graft material is then packed into the space and, finally, the mucoperiosteal flap is repositioned and the mucosa is sutured closed. Healing takes 6 months before implants are placed, but some surgeons will now place implants at the time of surgery if enough residual bone is available to support them. For successful implantation, most surgeons like to have bone that measures at least $5 \mathrm{~mm}$ in width and $7 \mathrm{~mm}$ in height and the sinus lift procedure attempts to provide at least this amount of bone. ${ }^{6}$

If the residual alveolar bone height is 3 to $6 \mathrm{~mm}$, a crestal approach to lifting the sinus lining and placing $8 \mathrm{~mm}$ implants may lead to less complications than a lateral window approach and placing implants at least $10 \mathrm{~mm}$ long. However, a shorter implant can be an easy and feasible alternative. Short implants (5 $\mathrm{mm}$ ) can be successfully loaded in maxillary bone with a residual height of 4 to $6 \mathrm{~mm}$, but their long-term prognosis is unknown. ${ }^{7}$

Various bone graft materials may be used. Initially, autogenous bone harvested from the tuberosity or the iliac crest was the graft material of choice because of its innate biocompatibility. Subsequently, the use of allografts, such as frozen bone, freeze-dried bone and demineralized freezedried bone became more common because their use eliminated donor site surgery.

When placing an implant in the anterior maxilla, care should be taken to avoid perforating the nasopalatine canal if the osteotomy site is in close proximity to the canal. ${ }^{3}$

\section{Mandibular Arch}

The most important anatomical consideration while placing an implant in the mandibular arch is the location of the of the inferior alveolar canal which contains the neurovascular bundles. Iatrogenic abuse of the vital structures like inferior alveolar nerve and artery can result into loss of sensation, altered sensation, pain, excessive bleeding, etc. following implant placement. Hence, it is important to determine the location as well as the configuration of the mandibular canal prior to implant placement. ${ }^{8}$

The location of the mandibular canal radiographically had been classified as:

- High - within $2 \mathrm{~mm}$ of the apices of the first and second molars

- Intermediate

- Low

- Other variations - duplication or division of the canal, partial or complete absence of canal, lack of symmetry. ${ }^{8}$ A study conducted by Heasman ${ }^{9}$ stated that in $68 \%$ of the cases, the mandibular canal traversed through the intermediate zone between the mandibular root apices and the inferior border of the mandible. In a dentate individual, the distance between the root apices of first and second molars and the upper border of the mandibular canal ranges from 3.5 to $5.4 \mathrm{~mm}$. However, once the teeth are lost, the residual alveolar bone undergoes varying degree of resorption and atrophy.

Levine et $\mathrm{al}^{10}$ conducted a study wherin they measured the distance between the edentulous alveolar crest and the superior aspect of the mandibular canal and concluded that the canal lied approximately. $17.4 \mathrm{~mm}$ inferior to the alveolar crest. However, this distance can vary and hence must be assessed in each case prior to implant placement.

The location of the mandibular canal is subjected to variation even in the horizontal plane.

Kim et al $^{11}$ classified the location of the mandibular canal in the buccolingual location into three types:

- Type 1: Canal follows the lingual cortical plate at the mandibular ramus and body (70\%).

- Type 2: Canal follows the middle of the ramus behind the 2nd molar and the lingual plate passing through the 2nd and the 1st molars (15\%).

- Type 3: Canal follows the middle or the lingual 1/3rd of the mandible from the ramus to the body (15\%).

Anatomical challenges, such as resorbed mandibular ridges and highly placed mandibular canal must be taken care of prior to implant placement through procedures such as ridge augmentation, bone grafts and transpositioning of the inferior alveolar nerve and artery. ${ }^{1}$

Presence of a large mandibular tori may give a false impression of the amount of available bone as well as hinder the outline of the mandibular canal. ${ }^{12}$

Complications in the anterior mandible may arise due to implant impinging on the mental nerve or an inferior 
perforation of the cortical plate. Hence, the amount of bone resorption and the location of the mental nerve in the interforaminal region of the anterior mandible should be considered prior to implant placement. 25 to $38 \%$ of cases present with the mental foramina located coronal to the premolar apex. ${ }^{12,13}$

Less frequent anatomical considerations include the anterior looping of the mental nerve, accessory mental formina and bifid mandibular canals. ${ }^{12}$

\section{Off-axis Implant Placement ${ }^{14}$}

Implants placed at positions off the vertical axis have been referred to as 'tilted implants' or 'off-axis fixtures.' These may be placed to avoid various anatomical structures or to eliminate the need for bone grafting and nerve repositioning procedures. Off-axis loading of the implants results in stress on the implants and the surrounding bone, but studies have found these stresses to be within the physiological limit.

Krekmanov et $\mathrm{al}^{14}$ recommended that posterior tilting of the distal implants in either arch may reduce cantilever length hence providing better load distribution. However, implants placed off-axis usually require angle-corrected abutments.

Rosén et $\mathrm{al}^{14}$ followed implants in the maxilla for 8 to 12 years that were tilted to avoid grafting procedures. They concluded that this was a successful alternative procedure to more resource-demanding techniques such as bone grafting.

Krennmair et al ${ }^{14}$ studied 62 patients with mandibular overdentures and analyzed the various angles of the implants for optimal restoration. They concluded that sagittal mandibular inclination should be attributed more importance than axial loading of implants.

Aparicio et al ${ }^{14}$ followed fixed implant bridges supported by both axial and tilted implants for 21 to 87 months postinsertion and concluded that the use of tilted implants is an effective and safe alternative to the maxillary sinus floor augmentation procedure.

\section{Implant Placement in Growing Individuals}

The dynamic relationship of dental and craniofacial development to the use of dental implants in a growing patient must be understood before the treatment is initiated. Maxillary and mandibular skeletal and dental growth results in dramatic changes in all three dimensions during active growth.

Experimental evidence and the behavior of ankylosed teeth suggest that an osseointegrated object remains stationary in the bone surrounding it and does not move or adapt to bone remodeling. Growth changes may result in the burying or loss of implants. Hence, implants placed in the early mixed dentition may lack the proper occlusion and lead to unesthetic situations particularly in the anterior segment. They may disturb the normal growth or may have to be replaced. Implants placed during late puberty or early adulthood have the best chance for long-term usefulness. For patients with normal facial profile, the implant placement should be postponed until the growth is complete. When possible, placement should be delayed until age 15 for females and 18 for males. However, placememt of implant may be indicated in children with hereditary anhidrotic ectodermal dysplasia (HAED), alveolar clefts, trauma and tumor resection. ${ }^{15}$

Mandibular growth in males continues up to the age of 20 to 30 years. In the long face type, frontally placed implants in the mandible tend to become more vestibularly placed due to growth changes. In the short facial type, there is an increased mesial drift such that implants in the front become lingual to natural dentition and the vertical growth in the premolar and molar areas lead to infraocclusion of implants. Danny Heij ${ }^{16}$ found that teeth show spontaneous mesial drift such that a medial movement of $5 \mathrm{~mm}$ is seen in the area between canine and first molar between the age of 10 and 21 years. Since implants lack such a drift, there is asymmetry seen in the arch, if the implants were placed laterally. On the other hand, if the implants were placed frontally, they would appear palatal to the natural teeth. Hence, position of the implants when placed adjacent to natural teeth is important, as implant do not exhibit any compensatory positional changes vertically or labiolingually.

According to studies by Ranly et al, ${ }^{16}$ implants placed in the maxillary anterior region at the age of 7 years will become $10 \mathrm{~mm}$ apical to the neighboring tooth. Movement of the tooth in maxilla with short facial types is usually in the horizontal direction. This makes the implants placed in the anterior segment to be more palatal to the adjacent teeth.

Thilander and Odman in their study have stated that implant placement in anterior maxilla should be delayed until the cessation of growth is observed. ${ }^{16}$

\section{Implants in Medically Compromised Patients}

Dental clinicians are confronted with an increasing number of medically compromised patients who require implant surgery for their oral rehabilitation. A few commonly encountered are mentioned below.

Diabetes: Diabetic patients show delayed wound healing, increased alveolar bone loss, increased periodontal disease, and increased inflammatory tissue destruction, all potentially complicating factors when placing implants. Also, bone and mineral metabolism are altered in diabetics, possibly 
interfering with the integration process. However, several studies have shown success with dental implants in patients with controlled diabetes.

Osteoporosis: Osteoporosis is the loss of bone mass and density throughout the body, including the jaws. Bone metabolism is impaired and thus, theoretically, osseous integration may be more difficult to achieve. Osteoporosis frequently occurs in postmenopausal women. August et al ${ }^{17}$ examined jaw differences in pre- and postmenopausal women and found more failures in postmenopausal women with maxillary implants, but not mandibular implants. The authors found that postmenopausal women not taking hormone replacements had the highest failure rates.

Xerostomia: There are numerous pathologic conditions that are accompanied by reduced salivary flow, e.g. after therapeutic head and neck irradiation , autoimmune diseases such as Sjögren's syndrome, systemic lupus erythematosus, progressive systemic sclerosis, primary or secondary amyloidosis, sarcoidosis, infectious diseases such as HIV and hepatitis $\mathrm{C}$ or diabetes mellitus. To date, there have been only a few reports on the use of implants in patients with xerostomia. These case reports indicate that these patients can be successfully treated with osseointegrated implants. However, prior to implant placement, the underlying cause of the xerostomia needs to be properly diagnosed and treated. ${ }^{17}$

\section{Implant Selection}

Appropriate diagnosis and a thorough treatment plan aids in the selection of the implant. It is imperative that an appropriate implant design and dimension is chosen for a given site, so that it provides the most ideal restorative platform both functionally and esthetically. To meet the market and the treatment requirements, implants representing a variety of designs and sizes have been introduced to the dental profession in the recent times. As implant choices have proliferated and esthetic expectations have risen, the task of selecting the appropriate implant for each site has become increasingly important.

A common axiom has been to place an implant as long as possible because the larger implants offer a greater boneto-implant interface and a wider prosthetic platform, increasing stability of prosthetic restorations. The available bone is greater in the anterior region of the mouth, especially the anterior mandible. But in the posterior regions, the available bone height is usually less and the implant cannot engage the dense opposing cortical bone either because it does not exist, as in the posterior maxilla or its beyond the anatomical limits, as in the mandible due to the presence of the neurovascular bundle. Placement of longer implants in these regions requires advanced surgical procedures, such as bone grafting and nerve repositioning procedure which adds to the complications. Hence, it is often not indicated, especially when other implant options are available. ${ }^{18}$

Shorter implants provide with a much less complex and invasive treatment option in clinical sites which require prior adjunctive procedures, such as ridge augmentation, grafting, sinus elevation or nerve repositioning. Besides, they need less bone removal as compared to longer implants and hence are less traumatic. Shorter implants can also be placed in sites where previous bone graft resorption has occurred. ${ }^{19}$

Although, studies have recommended $7 \mathrm{~mm}$ as a minimum requirement for implant length ${ }^{20}$ implants as short as $6 \mathrm{~mm}$ are now available and successfully placed. Their high success rate can be attributed to osseointegration, macrogeometric design of the implant and the distribution of forces.

Tawil et $\mathrm{al}^{21}$ in their study on shorter implants with a crown to implant ratio of less than 1 and greater than 2 , stated that short implants were a practicable option as long as the force orientation and load distribution were favorable.

Implant diameter is another factor to be considered while selecting an implant. The wide diameter implants have surgical, loading as well as prosthetic advantages. Wide diameter implants provide with greater surface area and hence are highly beneficial when placed in patients with parafunctional habits, increased crown height, increased masticatory dynamics in the posterior regions of the mouth. Besides, the wide diameter of the implants can help compensate for the shorter length of the implant placed in the posterior region due to anatomical constraints, if the force orientation and distribution is favorable. However, abundant bone volume is necessary for placement of wide diameter implants. ${ }^{22}$

Winkler et $\mathrm{al}^{23}$ in their study concluded that implants with diameter ranging from 3 to $3.9 \mathrm{~mm}$ showed lesser survival rate when compared to implants with diameter ranging from 4 to $4.9 \mathrm{~mm}$.

The ideal implant diameter most often corresponds to the width of the missing natural tooth $2 \mathrm{~mm}$ below the CEJ. Besides, an implant should at least be $1.5 \mathrm{~mm}$ away from the adjacent natural tooth whenever possible. The distance between two adjacent implants should be at least $3 \mathrm{~mm}$. Generally, anterior mandible has enough space for the placement of 4 to 6 implants. ${ }^{23}$ Whenever in doubt, it is wise to select an implant with a smaller diameter than the voted diameter. Implant with a minimum diameter of $4 \mathrm{~mm}$ is a must when placed in the posterior maxilla. 


\section{DISCUSSION}

The success of implant therapy depends on how well the implant is osseointegrated. Osseointegration has been defined as 'The apparent direct attachment or connection of osseous tissue to an inert, alloplastic material without intervening connective tissue'. Although implant therapy is the most viable treatment modality currently in the world of dentistry to replace the missing teeth, it is not without potential problems. A substantial number of implants do not integrate or fail to survive for long-term function. Hence, placement of implants should be undertaken only following thorough diagnosis and treatment planning. The phase of diagnosis and treatment planning should include a careful and detailed examination of the surgical site to identify any variations from normal in the anatomic structures of the maxilla and the mandible. One should make use of the available medical imaging modalities like CT and MRI, as they provide accurate information regarding the topography and location of the anatomical structures. They also provide information regrading the quality and quantity of the available alveolar bone which is essential to gauge the required implant dimensions, number as well as the position. Appropriate implant selection ensures achievement of predictable results.

Mandibular endosseous implant, if impinges on the neurovascular bundle that lies in inferior alveolar canal, can cause pain, paresthesia, numbness and excessive bleeding in case of injury to the artery. In the posterior maxilla, perforation of the sinus lining may occur, if the implant length is more than desired, leading to implant failure.

The length and diameter of the implant must be selected keeping in mind the available height and width of the bone. If the available bone is too little, advanced surgical procedures such as ridge augmentation, bone grafting, sinus elevation and nerve repositioning may be done. Shorter implants are a tangible option in clinical situations with insufficient available bone and should be considered whenever suitable.

\section{CONCLUSION}

The existing anatomical configuration is unique for each case and it dictates the selection and placement of the implant.

- The softer the bone, the longer the implant requirements.

- Greater the masticatory load, longer the implant requirements.

- Ideal implant length should be 12 to $16 \mathrm{~mm}$.

- Short implant length may be planned for anterior mandible.
- Wider implants, ranging from 3 to $6 \mathrm{~mm}$ may be placed in clinical situation with greater forces and less dense bone.

- Proper implant spacing and angulation should not be neglected.

\section{REFERENCES}

1. Pullen G, Debenham C. Short implants: Reality and predictability. Acad Dent Therap Stomato 2011 Jan:1-15.

2. Wood MR, Vermilyea SG. A review of selected dental literature on evidence-based treatment planning for dental implants: Report of the Committee on Research in Fixed Prosthodontics of the Academy of Fixed Prosthodontics. J Prosthet Dent 2004;92: 447-62.

3. Khoury F. Augmentation of the sinus floor with mandibular bone block and simultaneous implantation: A 6-year clinical investigation. Int J Oral Maxillofac Implants 1998;14:557-64.

4. Raghoebar GM, Vissink A. Treatment for an endosseous implant migrated into the maxillary sinus not causing maxillary sinusitis: Case report. Int J Oral Maxillofac Implants 2003;18(5):74.

5. Chappuis V, Suter VG, Bornstein MM. Displacement of a dental implant into the maxillary sinus: Report of an unusual complication when performing staged sinus floor elevation procedures. Int J Periodontics Restorative Dent 2009;29(1): 81-87.

6. Abrahams JJ, Hayt MW, Rock R. Sinus lift procedure of the maxilla in patients with inadequate bone for dental implants. AJR Am J Roentgenol 2000 May;174:1289-92.

7. Esposito M, Grusovin MG, Rees J, Karasoulos D, Felice P, Alissa R, et al. Effectiveness of sinus lift procedures for dental implant rehabilitation: A Cochrane systematic review. Eur J Oral Implantol 2010 Spring;3(1):7-26.

8. Juodzbalys G, Wang HL, Sabalys G. Anatomy of mandibular vital structures. Part I: Mandibular canal and inferior alveolar neurovascular bundle in relation with dental implantology. J Oral Maxillofac Res 2010 (Jan-Mar);1(1):e2.

9. Heasman PA. Variation in the position of the inferior dental canal and its significance to restorative dentistry. J Dent. 1988 Feb;16(1):36-39.

10. Levine MH, Goddard AL, Dodson TB. Inferior alveolar nerve canal position: A clinical and radiographic study. J Oral Maxillofac Surg 2007 Mar;65(3):470-74.

11. Kim ST, Hu KS, Song WC, Kang MK, Park HD, Kim HJ. Location of the mandibular canal and the topography of its neurovascular structures. J Craniofac Surg 2009 May;20(3): 936-39.

12. Juodzbalys G, Wang HL. Guidelines for the identification of the mandibular vital structures: Practical clinical applications of anatomy and radiological examination methods. J Oral Maxillofac Res 2010 (Apr-Jun);1(2):e1.

13. Juodzbalys G, Wang HL, Sabalys G. Anatomy of mandibular vital structures. Part II: Mandibular incisive canal, mental foramen and associated neurovascular bundles in relation with dental implantology. J Oral Maxillofac Res 2010 (Jan-Mar);1(1):e3.

14. Kurtzman GM, Dompkowski DF, Mahler BA, Howes DG. Offaxis implant placement for anatomical considerations using the co-axis implant. Inside Dentistry 2008;12(5):96-102.

15. Oesterle LJ, Cronin RJ Jr, Ranly DM. Maxillary implants and the growing patient. JOMI 1993Apr;377-86. 
16. Prasad DA, Prasad DK. Effect of implant placement in growing adults on cranioifacial development. A literature review. J Dent Imp 2012;2(2):97-102.

17. Beikler T, Flemmig TF. Implants in the medically compromised patient. Crit Rev Oral Biol Med 2003;14(4):305-16.

18. Oikarinen K, Raustia AM, Fartikainen M. General and oral local contraindications for endosteal implants: An epidemiological panoramic radiographic study in 65-year-old subjects. Comm Dent Oral Epidemiol 1995;23:114-18.

19. Misch C. Dental implant complications: Etiology, prevention and treatment (1st ed). Wiley- Blackwell 2010:57-62.

20. Wennstrom JL, Bengazi F, Lekholm U. The influence of the masticatory mucosa on the peri-implant soft tissue condition. Clin Oral Implants Res 1994;5:1-8.

21. Tawil G, Aboujaoude N, Younan R. Influence of prosthetic parameters on the survival and complication rates of short implants. Int J Oral Maxillofac Implants 2006 Mar-Apr;21(2): 275-82.

22. Misch CE. Contemporary implant dentistry (3rd ed). St Louis: Mosby, Inc. 2008:168-72.
23. Winkler S, Morris HF, Ochi S. Implant survival to 36 months as related to length and diameter. Ann Periodontol 2000;5: 22-31.

\section{ABOUT THE AUTHORS}

\section{Krishna Prasad (Corresponding Author)}

Professor and Head, Department of Prosthodontics, AB Shetty Dental College, Nitte University, Mangalore, Karnataka, India, Phone: 09448549831, e-mail: drkrishnaprasadd@yahoo.in

\section{Manoj Shetty}

Professor, Department of Prosthodontics, AB Shetty Dental College Nitte University, Mangalore, Karnataka, India

\section{Divya Rajan Mehra}

Postgraduate Student, Department of Prosthodontics, AB Shetty Dental College, Nitte University, Mangalore, Karnataka, India 